\title{
EL CONFLICTO SOCIOCOGNITIVO COMO INSTRUMENTO DE APRENDIZAJE EN CONTEXTOS COLABORATIVOS
}

\section{THE SOCIOCOGNITIVE CONFLICT AS A LEARNING TOOL IN COLLABORATIVE CONTEXTS}

\author{
Nadia Soledad Peralta* Néstor Daniel Roselli* ${ }^{* *}$ yna Borgobello***
}

\author{
*Doctora en Psicología. Becaria del Consejo Nacional de Investigaciones Científicas y Técnicas \\ (CONICET). E-Mail: nperalta@irice-conicet.gov.ar \\ **Doctor en Psicología. Miembro de la Carrera del Investigador Científico del Consejo Nacional de \\ Investigaciones Científicas y Técnicas (CONICET). E-Mail: roselli@irice-conicet.gov.ar \\ ***Doctora en Psicología. Becaria del Consejo Nacional de Investigaciones Científicas y Técnicas \\ (CONICET). E-Mail: borgobello@irice-conicet.gov.ar \\ Instituto Rosario de Investigaciones en Ciencias de la Educación (IRICE) \\ 27 de Febrero 210bis, (2000) Rosario, República Argentina.
}

\section{RESUMEN}

El conflicto sociocognitivo (CSC) ha sido tradicionalmente estudiado por medio de diseños experimentales de investigación como promotor del desarrollo en niños pequeños. En la actualidad, existe una línea de investigación que continúa esta orientación llevando el concepto al contexto del aprendizaje formal, dando lugar a diseños más variados. Sin embargo, muy pocas investigaciones hacen del CSC el eje de sus trabajos.

El estudio que se informa buscó incentivar en díadas de estudiantes universitarios el CSC en el aprendizaje colaborativo de conceptos.

Con el objetivo de evaluar las producciones escritas que los alumnos realizaban durante el aprendizaje y el efecto de aprendizaje logrado, se constituyeron tres grupos: uno experimental, formado por díadas colaborativas que fueron intervenidas por el investigador y dos grupos control (uno constituido por díadas que también trabajaron colaborativamente pero sin intervención y otro, por sujetos que trabajaron solos y que tampoco fueron intervenidos).

La muestra estuvo constituida por 120 sujetos, 60 alumnos de ciencias exactas (Física) y 60 alumnos de ciencias sociales (Psicología). Todos ellos eran estudiantes de primer año.

Se elaboraron cuestionarios y textos y se trabajó con un diseño de tipo experimental 'pre-test individual / intervención sobre la colaboración sociocognitiva / pos-test individual', estando el incentivo del CSC a cargo de un investigador que garantizaba la simetría de la participación, la evaluación mutua y la explicitación de las diferencias cognitivas.

Los resultados muestran que el incentivo del CSC es altamente eficaz en relación a las dos variables dependientes estudiadas: el aprendizaje logrado y la producción escrita; un efecto no esperado fue la mayor homogeneidad de los aprendizajes en las díadas intervenidas.

Palabras clave: Conflicto sociocognitivo; Aprendizaje colaborativo; Aprendizaje universitario; Interacción colaborativa.

\section{ABSTRACT}

Socio-cognitive conflict (SCC) has been defined as the exchange of different points of view 
when two or more people are working in a task together. It is cognitive because the points of view are intellectually supported and social because it takes place during an interaction. The concept of SCC was developed in the Geneva School of Educational Social Psychology and has been traditionally studied as an important factor in children development. These original studies were made using an experimental methodology, with samples of children between 6 and 7 years old. The basic learning tasks evaluated were piagetian tasks of conservation, for example, with liquids, length, quantity, etc. Nowadays, there is a new research field which takes this approach to study the SCC concept in formal learning contexts. This gave place to more varied designs. However, not many designs take SCC as their central aspect. The present study focuses on developing the SCC in pairs of university students using a pretest / post test experimental design. The collaborative learning environment is particularly critical for SCC to take place. This scenario is appropriate for the development of SCC because when pairs are working together sharing the same goal, the interaction increases. In this study the intervention focused on three signs of SCC: symmetry, peer evaluation and the cognitive differences between students. Researchers regulated a 'symmetrical' participation encouraging both students to participate in a similar amount of times and tasks. 'Peer evaluation' takes place when both students express their agreement or disagreement with the other one's speech. Researchers also asked students to express their disagreements even if they were shown as non-verbal expressions to increase the cognitive differences between them. In other words, the intervention was not focused on teaching concepts (as professors usually do in their classes); the goal was to guarantee the interaction between the students themselves. In order to evaluate the effect of the intervention on learning and on the interaction processes, three groups of students were constituted. One of them was the experimental group which was constituted by pairs who received the intervention of a researcher. The other two groups were control groups: one of them was integrated by pairs of students who worked independently, and the other one, by participants who worked alone. The sample had 120 participants, 60 students of an exact science career and 60 of a social science career. All students were in the first year of university. Post test evaluations show that encouraging SCC is effective in academic learning. In the experimental group there was more symmetry in participation and homogeneity in the learning process. In addition the written productions in this group were more complete, coherent, and logically structured than the control groups' productions. Educational implications for university level are discussed. These kinds of results are important because they could be used as an instrument for the classroom organization. It is also important as an example to consider ways to develop (especially with the university students), skills like dialogue, mutual understanding, team work, cognitive explanation, peer evaluation, self-evaluation, and respect for peers' points of view. All these skills can be developed with teachers who are interested in increasing SCC in collaborative learning environments.

Key words: Socio-cognitive conflict; Collaborative learning; University learning; Collaborative interaction.

\section{INTRODUCCIÓN}

Investigar en el contexto de enseñanza aprendizaje implica desde la concepción en la que fue hecho este estudio, entender que el aprendizaje no es sólo un producto localizado en la mente de los estudiantes, sino que necesariamente involucra una serie de procesos sociocognitivos, tanto en su génesis como en su mantenimiento. Se trata de procesos sociocognitivos porque el aprendizaje de conceptos se produce y sostiene en situaciones que son sociales per se, es decir, que son de intercambio con otros.

En este sentido, el estudio que se informa puede entenderse como situado en la corriente de la Psicología de la Instrucción (Genovard \& Gotzens, 1990; González Cabanach, Barca Lozano, Escoriza Nieto \& González Pineda, 1996; González Calleja, 1996), rama de la Psicología de la Educación 
que enfoca el aprendizaje de contenidos específicos en sujetos particulares en un contexto dado, con el objetivo de mejorar las prácticas de enseñanza-aprendizaje.

Un tipo de interacción ampliamente estudiado en relación con el aprendizaje es la colaboración (Christen, 2009; Kapp, 2009; Remedios, Clarke \& Hawthorne, 2008; Ünalan, 2008). Es posible hablar de aprendizaje colaborativo cuando dos o más sujetos emprenden una tarea intelectual que se transforma en un objetivo común, siendo los diferentes participantes beneficiados con la elaboración conceptual construida a través del andamiaje mutuo (Beers, Boshuizen, Kirschner \& Gijselaers, 2007; Dillenbourg \& Traum, 2006; Roselli, 2007). Los entornos de aprendizaje colaborativo son óptimos para que se desarrollen situaciones de distinto tipo que benefician el aprendizaje (Peralta, 2010; Peralta \& Borgobello, 2009), especialmente el conflicto sociocognitivo (CSC).

Este último concepto es útil para explicar los cambios estructurales que tienen lugar en la dinámica de los procesos de aprendizaje y surge de investigaciones post-piagetianas de la Escuela de Psicología Social de Ginebra (Doise \& Mugny, 1991; Mugny \& Pérez, 1988).

Cuando dos personas trabajan en la resolución de un determinado problema, puede suceder que se produzca un desacuerdo en las opiniones que estos sujetos tienen al respecto. El obstáculo que representa el punto de vista del otro hace que el sujeto comience a tener en cuenta su existencia. Esta situación exige una elaboración cognitiva que debe contener las perspectivas de ambos sujetos y debe ser superadora de las posiciones individuales. A este proceso se lo denomina conflicto sociocognitivo (CSC - Gilly, 1988; Perrer-Clermont, 1984; Roselli, 2008; Zittoun, 1997).

En la actualidad existirían al menos dos líneas principales de investigaciones empíricas que toman como eje el CSC. En la línea original, la mayor parte de los estudios se centran en el CSC como promotor de cambio de una estructura operacional (en términos piagetianos) a la siguiente. Se trata de investigaciones experimentales con niños preoperatorios y operatorios sobre la adquisición de nociones lógicas, como por ejemplo, el concepto de cardinal numérico (Morales Martínez, 2002) y de conservación de la longitud (Araúja de Cunha, 2004), en tareas consideradas típicamente piagetianas.

Un caso que ejemplifica esta primera línea de investigación es un estudio realizado por Dias (2003) cuyo objetivo fue conocer la adquisición de la noción de conservación de la longitud y de la realidad parcialmente escondida, a través del CSC con un diseño experimental pretest-postest. Durante la intervención experimental los sujetos veían videos que mostraban a niños conservadores y no-conservadores realizando una prueba de conservación y luego se realizaban sesiones de aprendizaje con incentivo del CSC. La importancia de este estudio radicó en que los autores pudieron demostrar que los efectos del incentivo del CSC fueron positivos en las capacidades cognitivas estudiadas.

Otro estudio típico de la misma línea (Psaltis \& Duveen, 2007) se focalizó en la emergencia del reconocimiento de la conservación de líquidos en situaciones de interacción con pares formados por un niño/a conservador/a y un/a no-conservador/a. En el grupo experimental, la intervención consistió en la explicitación e incentivo del CSC en torno a la realización de la tarea de conservación de líquidos de Piaget, mientras que en el grupo control no hubo intervención. Los resultados de este estudio demostraron nuevamente que la explicitación y el incentivo del CSC permiten el progreso cognitivo de los niños.

Mientras que en la primera línea de investigación el eje pasa por estudios experimentales referidos a tareas de índole lógica, una segunda línea se centra en la utilización del CSC en el aprendizaje de conceptos propios de la enseñanza formal con sujetos de diferentes edades y diseños variados. Esta línea incluye trabajos que relacionan el CSC con el aprendizaje de conceptos de Física en los primeros años de la universidad (Yeounsoo, Lei \& Acar, 2006), variables 
cognitivo-emocionales y cambio conceptual en sujetos de 13 y 14 años (Sukjin, Scharmann, Taehee \& Hanjoong, 2005) y el concepto de flotación en adolescentes (Skoumios, 2008), entre otros.

En un estudio encuadrado en esta segunda línea de investigación, por ejemplo, se analizó el papel que cumplía el CSC en el aprendizaje de un tema ad hoc en díadas de estudiantes de escuela primaria dirigidas por pares y profesores (Almasi \& Gambrell, 1994). El análisis sociolingüístico de los datos reveló que en los debates dirigidos por pares prevalecen los conflictos con uno mismo, los estudiantes producen un discurso más elaborado y complejo, pueden reconocer y resolver el CSC; mientras que en los debates dirigidos por el profesor, hay un predominio del conflicto con el texto y mayor dificultad para reconocer los CSC y resolverlos.

En otra investigación se analizó, a partir de discusiones de estudiantes universitarios sobre un tema, cómo los objetivos de aprendizajes y de desempeño influyen en el modo de regulación del CSC (Darnon, Muller, Schrager, Pannuzzo \& Butera, 2006). Cuando los estudiantes se plantean objetivos de aprendizaje reconocen la competencia de la otra persona, tratando de entender cómo su punto de vista puede servir de complemento a su propia comprensión, arribando a una regulación sociocognitiva del CSC. En cambio, cuando las personas se proponen objetivos de desempeño, tratan de imponer su punto de vista y mostrar el error en el compañero llegando de esta forma a una regulación relacional del CSC.

En síntesis, las investigaciones sobre CSC pueden ser dividas en dos grandes líneas. La diferencia entre ambas se centra en que en la primera predominan estudios con niños realizando tareas lógicas; mientras que en la segunda, predominan estudios de sujetos realizando actividades de aprendizaje de conceptos en la educación formal. Por tanto, puede considerarse a la primera línea centrada en la dimensión sintáctica (resolución de problemas) y a la segunda enfocada en la dimensión semántica (aprendizaje de contenidos).
En términos generales, existen condiciones previas e indicadores de CSC ampliamente acordados. En primer término, para que el mismo tenga lugar, debe establecerse una relación simétrica entre los participantes, es decir, deben considerarse a sí mismos como equivalentes, pudiendo aportar ambos cognitivamente a la tarea. A partir de allí, es posible que se produzcan evaluaciones mutuas entre pares que lleven posteriormente a la explicitación de disidencias cuando éstas existan. Si bien estas características del CSC suelen aparecer naturalmente en el trabajo colaborativo, las mismas aumentan en frecuencia cuando se incentiva a los participantes en este sentido, algo ampliamente comprobado en los estudios reportados como así también en los clásicos del tema (Carugati \& Mugny, 1988; Doise, 1991; Parisi, 1988; Perret-Clermont, 1984; Roselli, 1999a).

El objetivo de este estudio en particular fue comparar aprendizajes individuales logrados por sujetos que aprendieron colaborativamente y por otros que realizaron un aprendizaje individual, testeando específicamente los efectos (logros de aprendizaje de contenidos y producciones escritas) de la intervención optimizadora del conflicto. El Cuadro 1 sintetiza las variables del estudio.

\section{MÉTOdO}

MUESTRA

La muestra estuvo constituida por 120 estudiantes universitarios de primer año, de los cuales 60 pertenecían a una carrera de ciencias exactas (Física) y 60 a una carrera de ciencias sociales (Psicología) (ver Cuadro 2). La muestra se dividió al azar en tres submuestras:

1.- Un grupo experimental (GE) formado por 24 sujetos que trabajaron colaborativamente en 12 díadas con incentivo del CSC.

2.- Un grupo control I (GC I) formado por 24 sujetos que realizaron colaborativamente la tarea en 12 díadas sin incentivo del CSC. 
3.- Un grupo control II (GC II) integrado por 12 sujetos que trabajaron en forma individual.

\section{MATERIALES}

Los temas de trabajo fueron seleccionados de manera que ningún sujeto tuviera conocimiento específico previo de los mismos. Para controlar tal condición, se elaboraron cuestionarios $^{1}$ destinados a evaluar los conocimientos previos de los sujetos (pretest).

Para evaluar los efectos del tratamiento experimental, se diseñó un cuestionario ${ }^{1}$ para cada disciplina que sirvió para medir el aprendizaje individual logrado por cada sujeto (postest), pudiéndose obtener un puntaje entre 0 y 100 . Los mismos fueron aplicados en forma idéntica a cada sub-muestra. El postest de Psicología constaba de 14 preguntas cerradas y el de Física, de 4 preguntas cerradas y una abierta.

En el caso de Psicología, el tema fue Los Tests Psicológicos. El material fuente consistió en un texto modularizado ${ }^{1}$ que los sujetos debían estudiar y al finalizar cada módulo sub-temático, debían redactar (individual o colaborativamente, según la condición) una síntesis del mismo. Se trataba básicamente de una tarea de comprensión lectora.

En Física, el tema fue Movimiento Relativo. En este caso, el material consistió en un texto ${ }^{1}$ que presentaba un diálogo ficticio sobre fenómenos físicos de la vida cotidiana relativos al concepto de movimiento. A continuación proponía una serie de actividades relacionadas con el mismo, que iban más allá de la simple comprensión lectora del texto ofrecido ya que implicaban un trabajo de elaboración conceptual (por ejemplo, proponer ejemplos propios).

1 Los protocolos pueden ser solicitados a los autores.

\section{Procedimiento}

Se utilizó un diseño de tipo pretest-postest. El pretest se aplicó de manera individual con el objetivo de descartar aquellos sujetos que tuvieran un conocimiento previo de la temática.

A cada díada del GC I se les indicó que debían estudiar y aprender colaborativamente el material y que debían realizar libremente las actividades indicadas, disponiendo de 90 minutos para ello.

A los sujetos del GC II se les brindó el mismo material para que aprendieran y realizaran las actividades en forma individual, disponiendo también de 90 minutos.

A las díadas del GE se les indicó que debían estudiar y aprender colaborativamente el material, pero durante toda la interacción participó un investigador cuya intervención buscaba incentivar el CSC. Dicha intervención se informa en el Cuadro 3.

Es importante destacar que la intervención no estaba basada en la explicación de conceptos o en la corrección de los errores que pudieran tener los estudiantes, sino en garantizar la interacción misma. En otras palabras, la intervención no implicó ningún tipo de enseñanza o de apuntalamiento conceptual.

Todos los investigadores que trabajaron con el GE fueron entrenados para realizar correctamente la intervención y que la misma fuese similar en todos los casos.

Al finalizar y en todas las condiciones, a cada sujeto se le entregó el postest para que lo realizara en forma individual.

\section{Resultados}

Los postests de ambas condiciones (Física y Psicología) fueron corregidos con una puntuación de 0 a 100 .

Los resultados obtenidos por los alumnos de Psicología fueron los esperados, ya que las díadas que recibieron incentivo del CSC (GE) obtuvieron mejores puntuaciones que las pertenecientes al GCI y a los individuos que trabajaron solos (GCII). Dichas puntua- 
ciones fueron las siguientes: el GE tuvo un promedio igual a $84.45(D E=13.88)$, el GCI, igual a $75.40(D E=14.76)$ y el GCII, igual a $73.51(D E=16.72)$.

La comparación mediante la prueba $t$ de Student de estas medias mostró diferencias estadísticamente significativas entre el GE y el GCI $(t=-2.187 ; p=.034)$ y entre el GE y el GCII $(t=2.082 ; p=.045)$. Es decir, la mayor diferencia se presentó entre el GE y el GCI y no se registraron diferencias significativas entre ambos GC (ver Tabla 1).

En el caso de los alumnos de Física, los resultados demostraron que los sujetos que participaron en las díadas que recibieron el incentivo de CSC (GE), obtuvieron mejores resultados que los que trabajaron en díadas sin incentivo del CSC (GCI) y más aún que los que trabajaron individualmente (GCII).

Específicamente, la prueba $t$ de comparación del promedio del GE $(M=82.71$; $D E=7.22$ ) con el promedio del GCII $(M=68.75 ; D E=10.47)$ dio valores que resultaron estadísticamente significativos $(p=.001)$. Se fijó un valor de intervalo de confianza de $95 \%$ para el efecto del tratamiento (incentivo de CSC).

En cuanto a la comparación de los promedios entre el GE $(M=82.71 ; D E=7.22)$ con respecto al GC I $(M=76.88 ; D E=$ $10.71)$, la prueba $t$ también dio valores estadísticamente significativos $(p=.033)$ (ver Tabla 1).

Estos resultados indican que el logro individual de aprendizaje de los sujetos que pasaron por una situación de aprendizaje colaborativo con incentivo del CSC es mayor al de los que no recibieron ese incentivo.

En cuanto a las producciones escritas, la puntuación obtenida podía oscilar entre 1 y 5. Los escritos fueron evaluados de acuerdo a los siguientes criterios: claridad y corrección epistémica (redacción precisa y conceptualmente correcta), originalidad de la exposición (no debían ser copia textual) y exhaustividad de contenido (alusión a la mayor cantidad de ideas principales contenidas en el texto). Dicha evaluación se realizó en base a la opinión coincidente de tres evaluadores expertos independientes.
En cuanto a las producciones escritas de los alumnos de Psicología, los resultados mostraron diferencias estadísticamente significativas entre GE y GCI (díadas sin incentivo) y entre GE y GCII (trabajo individual), siendo estas diferencias mayores en el segundo caso (ver Tabla 2).

En las producciones escritas de los alumnos de Física las medias de cada grupo fueron las siguientes: $\mathrm{GE} M=4.10(D E=.26)$; GCII $M=3.35(D E=.87)$ y GCI $M=3.92$ $(D E=.27)$

La prueba $t$ indicó diferencias significativas entre GE y GCI $(p=.029)$ y entre GE y GCII $(p=.009)$. También se encontraron diferencias entre GCI y GCII $(t=2.384$; $p=.034)$ (ver Tabla 2).

Estos resultados mostraron que las producciones de las díadas que recibieron incentivo del CSC fueron superiores a las producciones de las díadas que no recibieron incentivo del CSC y a las de los sujetos que trabajaron individualmente. Asimismo, como las pruebas estadísticas lo sugieren, en general las producciones realizadas por las díadas fueron superiores a las producciones de los sujetos que trabajaron individualmente. Cabe recordar que cuando se plantea la superioridad de las producciones escritas, se hace referencia a los criterios evaluados, tales como la calidad, la originalidad y la exhaustividad.

Un efecto encontrado no previsto inicialmente fue el de la mayor homogeneidad, en términos de aprendizaje individual de los sujetos que trabajaron colaborativamente con incentivo (GE), con respecto a los que lo hicieron sin incentivo (GCI). Esto fue verificado a través de un cálculo de homogeneidad del aprendizaje realizado sobre los puntajes de los postests. Se calcularon las medias y los desvíos estándar de los resultados de aprendizaje (postest) de cada una de las díadas del GE y GC I, obteniéndose 12 medias y 12 desvíos para cada grupo, es decir, la media y el desvío entre los puntajes de los sujetos de cada díada. Posteriormente, se calculó la media de los 12 desvíos de cada grupo, lográndose así una medida comparativa de la homogeneidad intra-díada (un va- 
lor más bajo del promedio de los desvíos indicaría mayor homogeneidad).

A partir de este cálculo pudo observarse que los sujetos que participaron en díadas que recibieron incentivo del CSC tuvieron resultados más homogéneos entre sí que los sujetos que trabajaron en díadas pero que no recibieron incentivo. Este resultado fue evidenciado porque, en el caso de los alumnos de Psicología, el promedio de los desvíos intra-díadas del GE (5.52) fue menor al promedio del desvío intra-díadas del GCI (9.91). Lo mismo sucedió en el caso de los alumnos de Física en el cual el promedio del GE fue igual a 3.12, mientras que el del GCI fue igual a 7.29.

\section{Discusión}

Tal como se planteó, el objetivo de esta investigación fue comparar los aprendizajes individuales logrados por los sujetos que aprendieron colaborativamente con los que lo hicieron en forma individual, testeando específicamente los efectos de la intervención optimizadora del CSC.

El valor de los resultados permite demostrar la importancia que la colaboración y el incentivo del CSC tienen para los aprendizajes. En este sentido, cabe señalar que el $\mathrm{CSC}$ se desarrolla privilegiadamente en contextos de aprendizaje colaborativo ya que en este tipo de aprendizajes el énfasis reside en el intercambio entre iguales y la confrontación de puntos de vistas, permitiendo así la creación de consensos que benefician a los sujetos involucrados en este tipo de situaciones.

El estudio permitió demostrar que el incentivo del CSC en situaciones de aprendizaje colaborativo promueve mejores aprendizajes y producciones escritas de mayor calidad. Esto pudo evidenciarse en los resultados de las díadas intervenidas en las que el investigador incentivaba la simetría en la participación, la evaluación mutua entre los estudiantes y la explicitación de las disidencias, quedando de esta manera de- mostrados los efectos de la variable independiente testeada, o sea de la intervención.

Como ya se dijo, hay que tener presente que la intervención en ningún caso significó apuntalamiento cognitivo, sino exclusivamente estímulo de la interacción colaborativa. En todo caso, podría hablarse de un andamiaje procedimental, pero esto está lejos de la noción tradicional de apuntalamiento, ya que no está en juego el ofrecimiento externo de ninguna guía u orientación facilitadora del conocimiento. Esto habla de un efecto constructivo de la colaboración, que no tiene que ver con ninguna tutoría o efecto enseñante.

En lo que hace a la colaboración no incentivada y a la producción escrita colaborativa tampoco puede hablarse de complementariedad o enseñanza recíproca entre los alumnos, ya que el pretest demostró desconocimiento generalizado del tema específico. En todo caso podría hablarse de concurrencia de perspectivas o puntos de vista diferentes (en función del conocimiento general disponible), pero esto es precisamente lo que sostiene la teoría del conflicto sociocognitivo: el valor de la colaboración no reside en la posibilidad de enseñanza recíproca ni en una sumatoria de saberes, sino en la posibilidad de acceder a una pluralidad de puntos de vista distintos, aunque sean erróneos o imperfectos.

Estos resultados son consistentes con los reportados en las dos líneas de investigación referidas (las que analizan el CSC en relación a tareas sintácticas y las que lo hacen en relación a tareas semánticas). En cuanto al aspecto semántico o de contenido epistémico los resultados demuestran que esta variable (hipotetizada como interviniente) no parece tener un efecto discriminante, ya que las tendencias son bastante coincidentes entre los alumnos de Psicología y de Física, tanto en lo que hace a los postests (aprendizaje individual - producto), como a las producciones escritas (proceso de aprendizaje).

Una conclusión no prevista fue la constatación de una mayor homogeneidad de los aprendizajes obtenidos por los sujetos que 
participaron en instancias colaborativas incentivadas. Esto habla de un acercamiento entre los sujetos desde el punto de vista cognitivo, estableciendo un direccionamiento individual en función de un patrón colectivo. Podría decirse que cuando el proceso de enseñanza es colaborativo, los sujetos quedan aproximados, aún en su aprendizaje individual, a una cierta norma cognitiva establecida durante la interacción.

En estudios posteriores estos hallazgos deberían relacionarse con un análisis centrado en el aspecto comunicacional propiamente dicho, o sea con el intercambio discursivo evidenciado en tales contextos de colaboración cognitiva. Esto permitiría detectar posibles patrones de comunicación relacionados con la optimización de los aprendizajes. Asimismo, sería importante diseñar investigaciones basadas en el estudio del CSC en situaciones naturales y vincularlo con el rendimiento académico de los estudiantes.

Por otro lado, pueden plantearse algunas implicancias educativas a partir de los resultados de este estudio. En primer lugar, la necesidad de fomentar clases con mayor intercambio entre pares y situaciones que contemplen el aprendizaje colaborativo, debido a que las aulas universitarias son escenarios típicos de clases altamente expositivas.

En este punto es necesario reconocer que las clases universitarias son habitualmente numerosas. Una manera de contrarrestar esta dificultad es elaborar materiales didácticos que fomenten el intercambio de puntos de vista divergentes y la evaluación mutua. Asimismo, este material podría estar diseñado de modo que los estudiantes tengan que hacer productos escritos conjuntos para tener la posibilidad de evaluar la colaboración.

$\mathrm{Si}$ bien las clases con pequeños grupos son ideales para que el docente realice el tipo de intervención didáctica que se hizo en esta investigación, esto no supondría que en clases de grupos numerosos sea imposible diseñar estrategias en este sentido.

La forma en que se produce habitualmente el diálogo en clases numerosas tiene dos direcciones: docente - alumnos o alumno docente. Estrategias pensadas en torno al concepto de CSC implicarían sumarle a la direccionalidad habitual de ese diálogo, otras direccionalidades posibles, como por ejemplo, alumno - alumno (Roselli, 1999b).

Como reflexión final es posible pensar que, además de los resultados de aprendizaje a corto plazo evaluados en este estudio mediante el postest, las intervenciones de este tipo podrían tener efectos a largo plazo. Es importante generar, especialmente en los estudiantes universitarios, habilidades tales como el diálogo, el entendimiento mutuo, el trabajo en equipo, la fundamentación cognitiva, la evaluación de pares, la autoevaluación y el respeto por la opinión del otro. Todas estas habilidades pueden adquirirse en clases en las que se fomente el aprendizaje colaborativo y el CSC. 
CUADRO 1

VARIABLES DEL ESTUDIO

\begin{tabular}{|l|l|l|l|}
\hline \multirow{2}{*}{ Variables } & Independiente & Situación de aprendizaje & $\begin{array}{l}\text { Individual } \\
\text { Colaborativo libre } \\
\text { Colaborativo con incentivo del CSC }\end{array}$ \\
\cline { 2 - 4 } & Interviniente & Contenido epistémico & $\begin{array}{l}\text { Psicología } \\
\text { Física }\end{array}$ \\
\cline { 2 - 4 } & Dependiente & \multicolumn{2}{|c|}{ Producciones escritas } \\
& \multicolumn{2}{|c|}{ Aprendizaje individual } \\
\hline
\end{tabular}

CUADRO 2

DISEÑO GENERAL DE LA INVESTIGACIÓN

\begin{tabular}{|c|c|l|c|}
\hline Grupos & Pretest & \multicolumn{1}{|c|}{ Sesión experimental } & Postest \\
\hline GE & Individual & Aprendizaje colaborativo con intervención & Individual \\
\hline GC I & Individual & Aprendizaje colaborativo sin intervención & Individual \\
\hline GC II & Individual & Aprendizaje individual & Individual \\
\hline
\end{tabular}


Peralta, Roselli y Borgobello

\begin{tabular}{|c|c|c|c|c|c|}
\hline \multirow{3}{*}{ 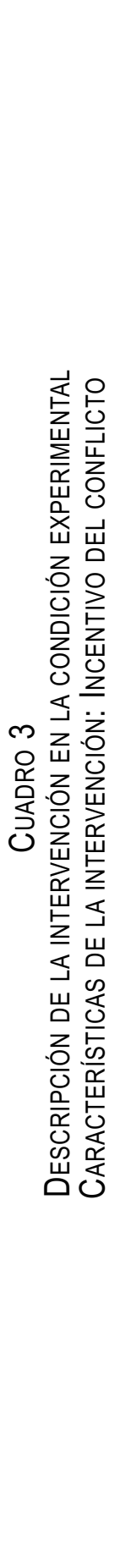 } & 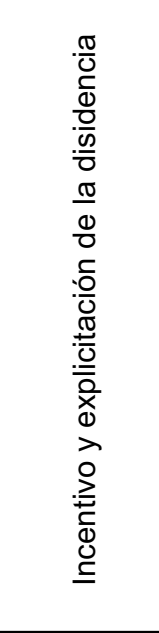 & 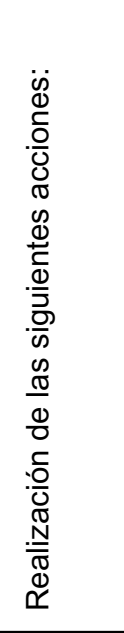 & 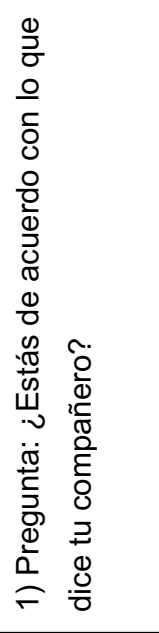 & 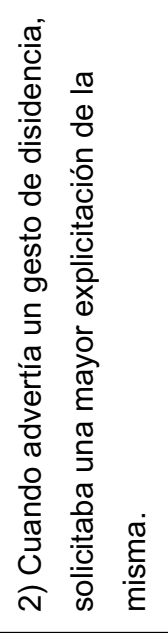 & \\
\hline & 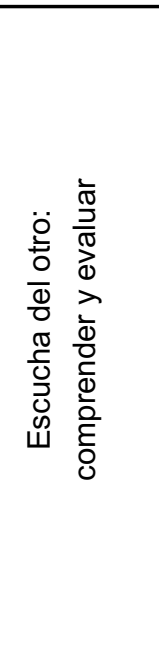 & 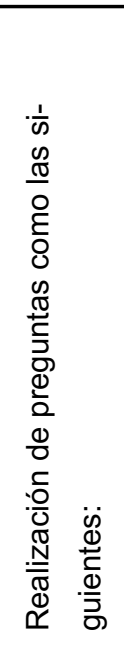 & 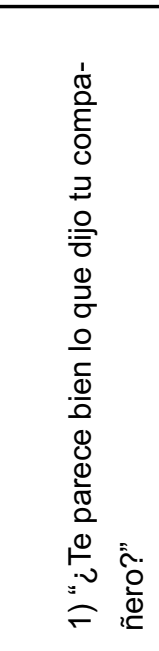 & 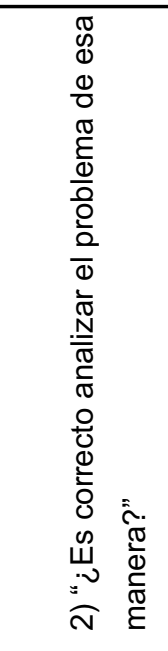 & 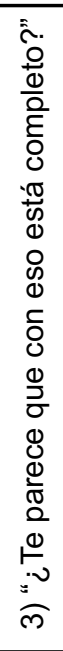 \\
\hline & 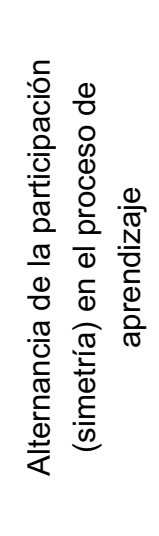 & 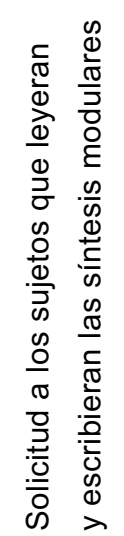 & 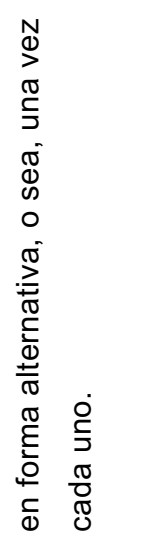 & & \\
\hline
\end{tabular}


TABLA 1

RESULTADOS OBTENIDOS EN LOS POSTESTS

\begin{tabular}{|c|c|c|c|c|c|c|c|c|}
\hline & \multicolumn{4}{|c|}{ Psicología } & \multicolumn{4}{|c|}{ Física } \\
\hline & GE & $\mathrm{GCl}$ & GE & GCII & GE & $\mathrm{GCl}$ & GE & GCII \\
\hline M & 84.45 & 75.40 & 84.45 & 73.51 & 82.71 & 76.88 & 82.71 & 68.75 \\
\hline$D$ & 13.88 & 14.76 & 13.88 & 16.72 & 7.22 & 10.71 & 7.22 & 10.47 \\
\hline$T$ & \multicolumn{2}{|c|}{$\begin{array}{c}t=-2.187 \\
g l=45.82 ; p=.034\end{array}$} & \multicolumn{2}{|c|}{$\begin{array}{c}t=2.082 \\
g l=34 ; p=.045\end{array}$} & \multicolumn{2}{|c|}{$\begin{array}{c}t=-2.212 \\
g l=40.31 ; p=.033\end{array}$} & \multicolumn{2}{|c|}{$\begin{array}{c}t=-4.150 \\
g l=16 ; p=.001\end{array}$} \\
\hline
\end{tabular}

TABLA 2

RESULTADOS OBTENIDOS EN LAS PRODUCCIONES ESCRITAS

\begin{tabular}{|c|c|c|c|c|c|c|c|c|}
\hline & \multicolumn{4}{|c|}{ Psicología } & \multicolumn{4}{|c|}{ Física } \\
\hline & GE & $\mathrm{GCl}$ & GE & GII & GE & $\mathrm{GCl}$ & GE & GII \\
\hline$M$ & 4.34 & 3.93 & 4.34 & 3.41 & 4.10 & 3.92 & 4.10 & 3.35 \\
\hline$D$ & .20 & .95 & .20 & .89 & .26 & .27 & .26 & .87 \\
\hline$T$ & \multicolumn{2}{|c|}{$\begin{array}{c}t=-2.070 \\
g l=25 ; p=.049\end{array}$} & \multicolumn{2}{|c|}{$\begin{array}{c}t=3.540 \\
g l=11.56 ; p=.004\end{array}$} & \multicolumn{2}{|c|}{$\begin{array}{c}t=-2.251 \\
g l=46 ; p=.029\end{array}$} & \multicolumn{2}{|c|}{$\begin{array}{c}t=-3.117 \\
g l=12 ; p=.009\end{array}$} \\
\hline
\end{tabular}




\section{REFERENCIAS BIBLIOGRÁFICAS}

Almasi, J.F. \& Gambrell, L.B. (1994). Socio-cognitive conflict in peer-led and teacher-led discussions of literature. Reading Research Report, 12, 1-40.

Araúja de Cunha, C.A. (2004). O papel do conflito sociocognitivo na aquisição da conservação de comprimento [The role of socio- cognitive conflict in acquiring conservation of length]. Psicologia em Estudo, 9(9), 111-118.

Beers, P.J., Boshuizen, H.P. Kirschner, P.A. \& Gijselaers, W.H. (2007). The analysis of negotiation of common ground in CSCL. Learning and Instruction, 17, 427-435. doi: 10.10 16/j.learninstruc.2007.04.002.

Carugati, F. \& Mugny, G. (1988). La teoría del conflicto sociocognitivo. En G. Mugny \& J.A. Pérez (Eds.), Psicología social del desarrollo [Developmental Social Psychology], (pp.7994). Barcelona: Anthropos.

Christen, A. (2009). Transforming the classroom for collaborative learning in the $21^{\text {st }}$ Century. Connecting Education \& Careers, 83(9), 2831.

Darnon, C., Muller, D., Schrager, S.M., Pannuzzo, N. \& Butera, F. (2006). Mastery and performance goals predict epistemic and relational conflict regulation. Journal of Educational Psychology, 98(4), 766-776. doi:10.10 37/0022-0663.98.4.766

Dias, F. (2003). Percepção social e cognição em situações de aprendizagem por conflito sociocognitivo [Social perception and cognition in learning situations by social-cognitive conflict]. Psico-USF, 8(1), 47-52. doi:10.1590/ S141382712 003000100007.

Dillenbourg, P. \& Traum, D. (2006). Sharing solutions: Persistence and grounding in multi- modal collaborative problem solving. Journal of the Learning Sciences, 15(1), 121-151. doi: 10.1207/s15327809j1s1501_9.

Doise, W. (1991). La doble dinámica social en el desarrollo cognitivo [Dual social dynamic in cognitive development]. Revista de Documentación Cientifica de la Cultura, 27, 12-20.

Doise, W. \& Mugny, G. (1991). Percepción intelectual de un proceso histórico. Veinte años de Psicología en Ginebra: Psicología social experimental [Intellectual perception of a historical process. Twenty years of Psychology in Geneva: Social experimental Psychology]. Revista de Documentación Científica de la Cultura, 124, 2-23.

Genovard, C. \& Gotzens, C. (1990). Psicología de la instrucción [Instructional Psychology]. Madrid: Santillana Aula XXI.

Gilly, M. (1988). Interacciones sociales y procedimientos en las construcciones cognitivas. En A.N. Perret-Clermont \& M. Nicolet (Dir.), Interactuar y conocer: Desafios y regulaciones sociales en el desarrollo cognitivo [Interacting and learning: Challenges and social regulations in cognitive development], (pp. 23-32). Buenos Aires: Miño y Dávila.

González Calleja, F. (1996). Metodología de investigación en psicología de la instrucción. En R. González Cabanach, A. Barca Lozano, J. Escoriza Nieto \& J.A. González Pineda (Eds.), Psicología de la instrucción. Volumen 1. Aspectos históricos, explicativos y metodológicos [Instructional Psychology. Volume 1. Historical, explanatory and methodological issues]. Barcelona: EUB.

González Cabanach, R., Barca Lozano, A., Escoriza Nieto, J. \& González Pineda, J.A. (1996). Psicología de la instrucción. Volumen 1. Aspectos históricos, explicativos y metodológicos [Instructional Psychology. Volume 1. 
Historical, explanatory and methodological aspects]. Barcelona: EUB.

Kapp, E. (2009). Improving student teamwork in a collaborative project-based course. Col- lege Teaching, 57(3), 139-143. doi:10.3200/ CTCH.57.3.139-143.

Morales Martínez, M.S. (2002). Aprendizaje del cardinal numérico: Conflicto cognitivo y cambio conceptual microgenético [Learning cardinal numbers: Cognitive conflict and microgenetic conceptual change]. Psicología Educativa, 8(2), 89-105.

Mugny, G. \& Pérez, J.A. (1988). Psicología social del desarrollo cognitivo [Social Psychology of cognitive development]. Barcelona: Anthropos.

Parisi, M. (1988). Niveles de organización cognitiva y permeabilidad al conflicto socio-cognitivo. En A.N. Perret-Clermont \& M. Nicolet (Dir.), Interactuar y conocer. Desafios y regulaciones sociales en el desarrollo cognitivo [Interacting and learning. Challenges and social regulations in cognitive development], (pp.33-43). Buenos Aires: Miño y Dávila.

Peralta, N. (2010). Teoría del conflicto sociocognitivo. De la operacionalidad lógica hacia el aprendizaje de conocimientos en la investigación experimental [Socio-cognitive conflict theory. From logic operability to knowledge acquisition in experimental research]. Revista Intercontinental de Psicología y Educación, $12(2), 121-146$.

Peralta, N. \& Borgobello, A. (2009). Estudio experimental del conflicto socio-cognitivo y del aprendizaje colaborativo en estudiantes universitarios de ciencias exactas. En M.C. Richaud y J.E. Moreno (Eds.), Recientes desarrollos iberoamericanos en investigación en Ciencias del Comportamiento [Latest developments in Latin American research in the
Behavioral Sciences] (Tomo 1, pp. 461-478). Buenos Aires: Ediciones CIIPME-CONICET.

Perrer-Clermont, A.N. (1984). La construcción de la inteligencia en la interacción social. Aprendiendo con los compañeros [The construction of intelligence in social interaction. Learning with Classroom Peers]. Madrid: Visor.

Psaltis, C. \& Duveen, G. (2007). Conservation and conversation types: Forms of recognition and cognitive development. British Journal of Developmental Psychology, 25(1), 79-102. doi:10.1348/026151005X91415.

Remedios, L., Clarke, D. \& Hawthorne, L. (2008). The silent participant in small group collaborative learning contexts. Active Learning in Higher Education, 9(3), 201-216. doi: 10.1177/1469787408095846.

Roselli, N.D. (1999a). La construcción sociocognitiva entre iguales [The socio-cognitive construction with classroom peers]. Rosario: IRICE.

Roselli, N.D. (1999b). El mejoramiento de la interacción sociocognitiva mediante el desarrollo experimental de la cooperación auténtica [Improving the socio-cognitive interaction with experimental developing of genuine cooperation]. Interdisciplinaria, 16(2), 123-151.

Roselli, N.D. (2007). El aprendizaje colaborativo: Fundamentos teóricos y conclusiones prácticas derivadas de la investigación empírica. En M.C. Richaud \& M.S. Ison (Comps.), Avances en investigación en Ciencias del Comportamiento en Argentina [Advances in Behavioral Science research in Argentina] (Tomo 1, pp. 479-498). Mendoza: Editorial de la Universidad del Aconcagua.

Roselli, N.D. (2008). La teoría del conflicto sociocognitivo y su aplicación al cambio conceptual [Socio-cognitive conflict theory and its 
application to conceptual change]. 12(ntes), 26, 13-14.

Skoumios, M. (2008). Socio-cognitive conflict processes in science learning: Benefits and limits. Journal of Baltic Science Education, 7(3), 165-174.

Sukjin, K., Scharmann, L., Taehee, N. \& Hanjoong, K. (2005). The influence of students' cognitive and motivational variables in respect of cognitive conflict and conceptual change. International Journal of Science Education, 27(9), 1037-1058.
Ünalan, H. T. (2008). The effectiveness of collaborative learning applications in art education. The Journal of International Social Research, 1(5), 869-879.

Yeounsoo, K., Lei, B. \& Acar, O. (2006). Students' cognitive conflict and conceptual change in physics by inquiry class. AIP Conference Proceedings, 818(1), 117-120.

Zittoun, T. (1997). Note sur la notion de conflit socio-cognitif [Note on the concept of sociocognitive conflict]. Cahiers de Psychologie, 33, 27-30.

Instituto Rosario de Investigaciones en Ciencias de la Educación (IRICE)

Consejo Nacional de Investigaciones Cientificas y Técnicas (CONICET) Universidad Nacional de Rosario (UNR). Rosario - Santa Fe República Argentina

Fecha de recepción: 25 de abril de 2012 Fecha de aceptación: 23 de julio de 2012 